\title{
Failure to perform assisted deliveries is resulting in an increased neonatal and maternal morbidity and mortality: An expert opinion
}

\author{
R C Pattinson, ${ }^{1} \mathrm{MD}$, FRCOG, FCOG (SA); V Vannevel, ${ }^{1} \mathrm{MD}$, MMed (O\&G) (KU Leuven, Belgium); D Barnard, ${ }^{1} \mathrm{MB}$ ChB, FCOG (SA), \\ MMed (O\&G); S Baloyi, ${ }^{2}$ MB ChB, CML, CRIA, Dip Obst (SA), FMAS, Dip MAS, FCOG (SA), MMed (O\&G), PGDip Fam Med, MSc, \\ PGDPH; G S Gebhardt, ${ }^{3}$ MB ChB, Dip Obst (SA), MMed (O\&G), FCOG (SA), MSc (Med Sci), PhD; K le Roux, ${ }^{4}$ MB ChB, MMedSci \\ (Uppsala), DA (SA), Dip Obst (SA); N Moran, ${ }^{5}$ BM BCh, MA, FCOG (SA); J Moodley, ${ }^{6}$ MB ChB, FRCOG, FCOG, MD

\begin{abstract}
${ }^{1}$ South African Medical Research Council/University of Pretoria Maternal and Infant Health Strategies Unit, Department of Obstetrics and Gynaecology, School of Medicine, Faculty of Health Sciences, University of Pretoria, South Africa

${ }^{2}$ Academic Head, Department of Obstetrics and Gynaecology, Faculty of Health Sciences, University of the Free State, Bloemfontein, South Africa

${ }^{3}$ Department of Obstetrics and Gynaecology, Faculty of Medicine and Health Sciences, Stellenbosch University and Tygerberg Hospital, Cape Town, South Africa

${ }^{4}$ Honorary Lecturer, Department of Family Medicine, Faculty of Health Sciences, Walter Sisulu University, Mthatha, Eastern Cape, South Africa; Honorary Lecturer, Primary Health Care Directorate, University of Cape Town, South Africa; Zithulele Hospital, Eastern Cape, South Africa

${ }^{5}$ Head of Clinical Department: Obstetrics and Gynaecology, KwaZulu-Natal Department of Health, South Africa; Honorary Lecturer, Department of Obstetrics and Gynaecology, Nelson R Mandela School of Medicine, College of Health Sciences, University of KwaZulu-Natal, South Africa

${ }^{6}$ Chair: National Committee for Confidential Enquiries into Maternal Deaths, South Africa
\end{abstract}

Corresponding author: V Vannevel (valerie.vannevel@up.ac.za, valerie.vannevel@gmail.com)

\begin{abstract}
The need to perform assisted vaginal delivery (AVD) has been regarded as self-evident. In high-income countries, rates of AVD range between $5 \%$ and $20 \%$ of all births. In South Africa, the rate of AVD is only $1 \%$. This has resulted in increased neonatal morbidity and mortality due to intrapartum asphyxia, and increased maternal morbidity and mortality due to a rise in second-stage caesarean deliveries. In this article, we address the possible causes leading to a decrease in AVD and propose measures to be taken to increase the rates of AVD and subsequently reduce morbidity and mortality.
\end{abstract}

S Afr Med J 2018;108(2):75-78. DOI:10.7196/SAMJ.2018.v108i2.12786

The need to perform assisted vaginal delivery has been regarded as self-evident. Textbooks state:

'Assisted vaginal delivery (AVD) offers the option of an operative procedure to safely and quickly remove the infant, mother and obstetrician from a difficult or even hazardous situation. When spontaneous vaginal delivery does not occur within a reasonable time, a successful AVD or operative vaginal delivery trial avoids caesarean delivery with its attendant uterine scar and implications for future pregnancy and avoids potential birth asphyxia from prolonged fetal and cord compression. ${ }^{[1]}$

'Forceps and ventouse deliveries are among the most common obstetric interventions. Sober judgement and a thorough understanding of the normal mechanism of labour and the instruments to be used are the keys to success. ${ }^{[}[2]$

There have been no randomised trials comparing caesarean delivery (CD) with AVD. The only review remotely applicable is one by Majoko and Gardener ${ }^{[3]}$ on the trial of instrumental delivery in theatre v. immediate $\mathrm{CD}$ for anticipated difficult assisted births. In this review they could find no studies. The Cochrane Library has a review on the choice of instruments for AVD by O'Mahony et al. ${ }^{[4]}$

Majoko and Gardener ${ }^{[3]}$ state: 'The majority of women have spontaneous vaginal births, but some women need assistance in the second stage with delivery of the baby, using either the obstetric forceps or ventouse extraction. Rates of instrumental vaginal delivery range from $5 \%$ to $20 \%$ of all births in high income countries. The majority of instrumental vaginal deliveries are conducted in the delivery room, but in a small proportion ( $2 \%$ to $5 \%$ ), a trial of instrumental vaginal delivery is conducted in theatre with preparations made for proceeding to CD'

A recent cross-sectional study on the use of assisted deliveries in 40 low- and middle-income countries in Latin America, sub-Saharan Africa and $\mathrm{Asia}^{[5]}$ showed that only $1 \%$ of patients were delivered using AVD (either vacuum or forceps). The primary obstacle to performing an AVD was lack of healthcare workers trained in the procedure.

The high incidence of AVD in high-income countries could be attributed to the presence of better-skilled healthcare workers and available equipment. However, a proportion of AVDs are likely to be due to the wide use of epidural analgesia in labour, which has been shown to increase the risk for AVD. ${ }^{[6]}$

\section{The current situation in South Africa}

AVD is performed during the second stage of labour, and the clinical indications are related to maternal and fetal wellbeing.

\section{Maternal indications}

- Failure to progress in the second stage of labour, for example owing to maternal exhaustion or occipitoposterior presentation. Signs of cephalopelvic disproportion (CPD) leading to obstructed labour must, however, be excluded. 
- Medical conditions where maternal pushing is contraindicated or should be minimised, such as severe hypertension, or cardiac or respiratory disease.

\section{Fetal indications}

- Fetal distress in the second stage of labour (without signs of CPD).

Data from the Birthplace in England Collaborative Groups study on perinatal outcomes of low-risk pregnancies ${ }^{[7]}$ show a perinatal mortality rate (PNMR) of $<1 / 1000$ births in this population. The assisted delivery rate was $13.5 \%$ (7.3\% ventouse and $6.2 \%$ forceps deliveries).

In South Africa (SA), data from the Perinatal Problem Identification Programme (PPIP) are analysed and reported in a Saving Babies report. The eighth report ${ }^{[8]}$ which analysed data from 2010 and 2011, shows a PNMR for babies weighing $\geq 1000 \mathrm{~g}$ of 25.6/1 000 . Intrapartum asphyxia and birth trauma was the major underlying cause of perinatal death, with a rate of 4.87/1 000 (when macerated stillbirths were excluded and fresh stillbirths and early neonatal deaths included). Data from 2012 and $2013^{[9]}$ show a PNMR of 24.8/1 000 for babies weighing $\geq 1000 \mathrm{~g}$, with intrapartum asphyxia and birth trauma still comprising one of the major causes of perinatal mortality with a rate of $4.26 / 1000$ births. These data show that 15 $20 \%$ of all perinatal deaths are due to intrapartum asphyxia and birth trauma (19.0\% in 2010 - 2011, 17.1\% in 2012 - 2013).

The assisted delivery rate in SA (reported in 2010 - 2011) was $0.52 \%$ for ventouse and $0.15 \%$ for forceps. The $\mathrm{CD}$ rate was $21 \%$. When the intrapartum asphyxia and birth trauma death rate was correlated with the ventouse delivery rate, there was a significant negative correlation of $r=-0.307, p=0.036$ (Fig. 1). A negative correlation means that the fewer assisted deliveries by ventouse were performed, the more intrapartum asphyxia and birth trauma deaths were observed in that district. This could be explained by good obstetric care leading to an increased use of AVD and a subsequently lower rate of perinatal deaths due to intrapartum asphyxia and birth trauma.

An overall assisted delivery rate of $<1 \%$ is too low and is probably due to loss of skill in performing AVD and/or lack of willingness to perform it, as well as lack of the necessary equipment. Even at the tertiary level there is lack of ability to perform AVD, as shown in Table 1: only three out of four doctors and midwives at a provincial

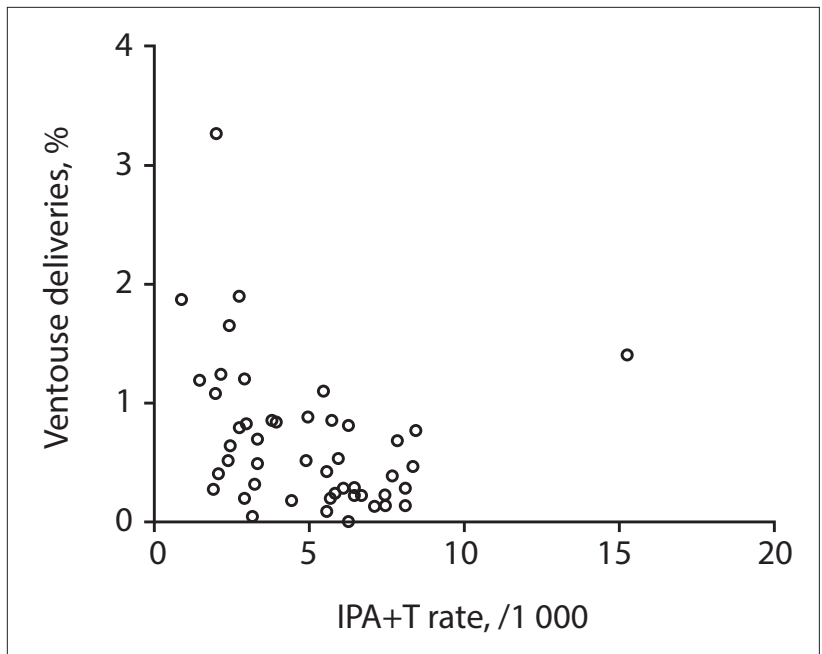

Fig. 1. Correlation between IPA + T rate and ventouse delivery rate, 2010 $2011{ }^{[8]}$ (IPA+T = intrapartum asphyxia and birth trauma rate. Each dot represents a district.) tertiary hospital could perform a ventouse delivery, whereas for forceps the figure is only two out of four.

AVD is a key activity to reduce perinatal deaths by reducing delay and is therefore an important function for community health centres (CHCs) in the correct circumstances. We have to distinguish between a clinic/midwifery obstetric unit and a district hospital with CD facilities. A midwife at a remote clinic should have only one indication for AVD: failure to progress during the second stage of labour with the fetal head on the perineum. This includes cases where there is maternal exhaustion or fetal distress. All advanced midwives should be able to perform at least a ventouse delivery. The fact that these skills are almost totally absent in the CHCs (Table 1) speaks for itself and must contribute to the high mortality rate due to intrapartum asphyxia and birth trauma. Patients with persistent occipitoposterior or other malpresentations where the head is not yet fully descended will be better off with a trial of AVD in a hospital where $\mathrm{CD}$ can be performed, rather than a failed rotational ventouse at the clinic and then transport to a hospital.

The problem of attaining and maintaining the right level of skill could explain the very low rates of AVDs. In a survey of clinicians involved in obstetrics in KwaZulu-Natal Province, SA, the vast majority indicated that they were more comfortable doing a CD than an AVD, and only 9\% used AVD after training (through the Essential Steps in Managing Obstetric Emergencies programme). ${ }^{[1]}$ The main reasons for this were lack of confidence, experience and supervision and fear of litigation. Lennox ${ }^{[12]}$ added words of warning: 'The decision to perform and successfully carry out an operative vaginal delivery may require a much higher level of judgement and skill than that involved in CD.'

The reasons why AVD has fallen out of favour in SA may include: - Prevention of mother-to-child transmission of HIV (PMTCT) guidelines advising against the use of instrumental delivery, particularly ventouse delivery

- A misconception that instrumental delivery increases the likelihood of brain injury for the baby

- A concern that conducting instrumental delivery may be a risk for subsequent litigation.

The subsequent rise in CDs has resulted in an increase in maternal deaths due to haemorrhage during or after $\mathrm{CD}$, which account for a third of all deaths due to obstetric haemorrhage in SA. ${ }^{[13]}$ A second-stage CD is more difficult to perform and associated with higher morbidity than CD in the first stage of labour due to haemorrhage, extended hospital stay, a greater risk of bladder trauma and unintended extensions of the uterine incision. ${ }^{[14]}$

In a prospective cohort study in Bristol, UK, the outcomes of mothers and neonates after delivery by second-stage CD were compared with the outcomes after AVD in theatre (anticipated difficult AVD). ${ }^{[15]}$ The patients delivered by CD had significantly more haemorrhage ( $>1000 \mathrm{~mL}$ ), a higher rate of prolonged hospital stay ( $>6$ days) and more neonatal intensive care unit admissions due to low Apgar scores and low umbilical artery $\mathrm{pH}$. The haemorrhage was also more severe if the CD was performed by a less skilled surgeon.

Expediting delivery by either CD or AVD is often done to avoid long-term neurological complications such as cerebral palsy. Several retrospective studies comparing neonatal morbidity after CD v. AVD have been published. Werner et al. ${ }^{[16]}$ found that forceps deliveries were associated with a significantly reduced risk of adverse neonatal neurological outcome (when regarding seizures and intraventricular and subdural haemorrhage as the morbidities most predictive of future neurodevelopmental deficits). Forceps delivery was also 
Table 1. Ability to perform assisted vaginal delivery in healthcare facilities in 12 districts in South Africa ${ }^{[10]}$

\begin{tabular}{|c|c|c|c|c|}
\hline Procedure & $\mathrm{CHC}(N=54), n(\%)^{*}$ & $\mathrm{DH}(N=63), n(\%)$ & $\mathrm{RH}(N=13), n(\%)$ & PT $(N=4), n(\%)$ \\
\hline Ventouse (by doctor or midwife) & $3(5.6)$ & $36(57.1)$ & $10(76.9)$ & $3(75.0)$ \\
\hline Forceps (by doctor or midwife) & $3(5.6)$ & $38(60.3)$ & $9(69.2)$ & $2(50.0)$ \\
\hline Ventouse by midwife & $2(3.7)$ & $12(19.0)$ & $3(23.1)$ & $1(25.0)$ \\
\hline Forceps by midwife & $1(1.9)$ & $9(14.3)$ & $2(15.4)$ & $1(25.0)$ \\
\hline
\end{tabular}

associated with significantly fewer 5-minute Apgar scores $<7$. A study by Towner et al. ${ }^{[17]}$ suggests abnormal labour to be a common risk factor for intracranial haemorrhage, as the rates did not differ between AVD and CD.

An additional benefit of AVD over CD is the cost benefit. ${ }^{[18]}$

There are very few data available on mother-to-child transmission (MTCT) of HIV with AVD. A large population-based surveillance study in the UK and Ireland (National Study of HIV in Pregnancy and Childhood $)^{[19]}$ has collected data on deliveries since 2008. Of the 9072 live births reported, 251 were assisted vaginal deliveries. One infant was reported to have acquired HIV. However, there were other significant risk factors that could have contributed to this transmission, such as poor maternal adherence to antiretroviral (ARV) therapy and possible breastfeeding. The Royal College of Obstetricians and Gynaecologists guideline on operative vaginal delivery ${ }^{[20]}$ states that blood-borne viral infections of the mother are no contraindication to operative vaginal delivery. One should, however, avoid difficult deliveries with an increased risk of fetal abrasion or scalp trauma, as this could increase vertical MTCT. The Cochrane review on choice of instruments for $\mathrm{AVD}^{[4]}$ states that theoretically any scalp injury could increase the risk of MTCT. Soft vacuum cups are associated with a lower risk of scalp injury, but have a higher failure rate than other types of vacuum. Metal vacuum cups seem to have a higher success rate than hand-held vacuums, but there were only three trials available for this comparison. Newer randomised trials have found no difference in success rates. ${ }^{[21,22]}$ Hand-held vacuums may be more easily available and require no other equipment.

A reduction in deaths of pregnant women and their infants is possible if skills in AVD and the culture of performing AVD are established. It is a misconception that AVD leads to birth asphyxia and maternal morbidity. In fact, it is the other way round: failure to do an assisted delivery is a major cause of birth asphyxia and CD-related maternal morbidity. To improve our quality of care for women in labour and their infants, we must improve the skills of performing assisted deliveries and increase the number of people able, willing and confident to do so.

\section{Requirements to increase appropriate use of AVD}

1. Standardised equipment

- Disposable ventouse at every delivery site. This should be in addition to an electrically operated vacuum machine, with re-usable cups.

- Wrigley's forceps at every hospital conducting deliveries.

\section{Training}

- A dedicated training course on managing the second stage of labour, including assisted deliveries, using good-quality mannequins and one-on-one tuition. This training must also include training on diagnosing the correct position of the fetal head. Such a course can be incorporated into the Essential Steps in the Management of Obstetric Emergencies (ESMOE) programme and should be part of all registrars' training in obstetrics and gynaecology.

- 'Current educational methodology promotes the use of skills and drills scenarios employing mannequins and models to teach clinical skills. For example, most postgraduate deaneries in the UK require evidence of such training as this forms part of the trainees' core logbook of clinical skills. Thus, methods to achieve, evaluate and maintain competence in AVD vaginal delivery need to be established ${ }^{[4]}$

3. Supportive environment for converting training into actual performance of instrumental delivery in the workplace

- Change in the PMTCT guideline: removing the guideline that instrumental deliveries should be avoided in HIV-infected women and stating that AVD can be performed in women who are HIV-positive, as long as there is a clear indication for instrumental delivery. In the absence of supporting scientific data, the benefit of AVD for both mother and neonate often outweighs the theoretical risk of vertical transmission after scalp injury. The indications for instrumental delivery should not differ according to HIV status. All HIV-positive women should be on ARVs during labour, which will minimise the risk of vertical transmission. Further care should be taken to avoid scalp injury during the procedure: the vacuum should be released prior to removal of the cup, and the site on the scalp where the cup was attached can be cleansed after removal of the cup and kept away from maternal secretions thereafter.

- A clear guideline from the South African Nursing Council stating that advanced midwives are expected to perform ventouse deliveries when indicated as part of their scope of practice, and must have performed this skill a minimum number of times (e.g. 5 times) as part of their logbook requirements.

- Emphasis on the need for all medical interns to have performed at least 3 ventouse deliveries as part of their logbook requirements.

- Emphasis on the need for registrars in family medicine to have performed at least 3 ventouse deliveries as part of their logbook requirements.

- Emphasis on the need for registrars in obstetrics and gynaecology to have performed at least 10 ventouse deliveries as part of their logbook requirements, including at least one directly observed and signed for by a supervising specialist.

- Circular to be sent to all maternity delivery facility chief executive officers, medical and nursing managers, and heads of obstetrics and gynaecology and paediatric departments explaining the essential need for women delivering at that facility to have access to instrumental delivery when indicated clinically, and explaining the likely benefits in terms of reduced morbidity and litigation. The circular should specify the categories of staff that are expected to have skills in conducting the procedure. 


\section{Conclusion}

Reviving the culture of performing AVD in SA is likely to reduce the current high rates of perinatal morbidity and mortality due to birth asphyxia. Furthermore, it will reduce maternal morbidity and mortality arising from second-stage $\mathrm{CD}$. This change in culture can only be achieved through a concerted effort by healthcare managers, maternity care educators and trainers, and by the clinical supervisors working in the labour ward.

Acknowledgements. We thank the National Department of Health for assistance in making this report possible. The report was submitted to and approved by the National Committee for Confidential Enquiries into Maternal Deaths and the National Perinatal Morbidity and Mortality Committee.

Author contributions. All authors contributed to this article.

\section{Funding. None.}

\section{Conflicts of interest. None.}

1. Lindow SW, Hayashi R. Assisted vaginal delivery. In: James D, Steer PJ, Weiner CP, Gonik B, Crowther CA, Robson SC. High Risk Pregnancy: Management Options. 4th ed. St Louis, Mo.: Elsevier Saunders, 2011:1245-1259.

2. Brand SSF. Forceps and ventouse delivery. In: Cronjé HS, Cilliers BF, Pretorius MS. Clinical Obstetrics: A South African Perspective. 3rd ed. Pretoria: Van Schaik, 2010:328-344.

3. Majoko F, Gardener G. Trial of instrumental delivery in theatre versus immediate caesarean section for anticipated difficult assisted births. Cochrane Database Syst Rev 2012, Issue 10. Art. No.: CD005545. anticipated difficult assisted births. Cochrane Dat
https://doi.org/10.1002/14651858.cd005545.pub3

4. O'Mahony F, Hofmeyr GJ, Menon V. Choice of instruments for assisted vaginal delivery. Cochrane Database Syst Rev 2010, Issue 11. Art. No.: CD005455. https://doi.org/10.1002/14651858.cd005455.pub2 5. Bailey PE, van Roosmalen J, Mola G, Evans C, de Bernis L, Dao B. Assisted vaginal delivery in low and middle income countries: An overview. BJOG 2017;124(9):1335-1344. https://doi.org/10.1111/14710528.14477

6. Anim-Somuah M, Smyth RMD, Jones L. Epidural versus non-epidural or no analgesia in labour. Cochrane Database Syst Rev 2011, Issue 12. Art. No.: CD000331. https://doi.org/10.1002/14651858. cd000331.pub3
7. Birthplace in England Collaborative Group. Perinatal and maternal outcomes by planned place of birth for healthy women with low risk pregnancies: The Birthplace in England national prospective cohort study. BMJ 2011;343:d7400. https://doi.org/10.1097/01.aoa.0000426077.71832.25

8. Pattinson RC. Saving Babies 2010 - 2011: Eighth Report on Perinatal Care in South Africa. Pretoria: Tshepesa Press, 2013

9. Pattinson RC, Rhoda N. Saving Babies 2012 - 2013: Ninth Report on Perinatal Care in South Africa. Pretoria: Tshepesa Press, 2014

10. Pattinson RC, Makin JD, Pillay Y, van den Broek N, Moodley J. Basic and comprehensive emergency obstetric and neonatal care in 12 South African health districts. S Afr Med J 2015;105(4):256-260. https://doi.org/10.7196/SAMJ.9181

11. Devjee A. A survey of health professionals on the current use of forceps/ventouse and skills for operative vaginal delivery. Obstet Gynaecol Forum 2015;25(3):37-39.

12. Lennox C. Difficult labour. In: Lawson JB, Harrison KA, Bergstrom S. Maternity Care in Developing Countries. London: RCOG Press, 2001:179-200.

13. Pattinson RC, ed. Saving Mothers 2011 - 2013: The Sixth Report of the National Committee for Confidential Enquiry into Maternal Deaths in South Africa. Pretoria: Government Printer, 2014.

14. Allen VM, O'Connell CM, Baskett TF. Maternal and perinatal morbidity of caesarean delivery at full cervical dilatation compared with caesarean delivery in the first stage of labour. BJOG 2005;112(7):986cervical dilatation compared with caesarean delivery in
990 . https://doi.org/10.1111/j.1471-0528.2005.00615.x

15. Murphy DJ, Liebling RE, Verity L, Swingler R, Patel R. Early maternal and neonatal morbidity associated with operative delivery in second stage of labour: A cohort study. Lancet 2001;358(9289):1203-1207. https://doi.org/10.1016/s0140-6736(01)06341-3

16. Werner EF, Janevic TM, Illuzzi J, Funai EF, Savitz DA, Lipkind HS. Mode of delivery in nulliparous women and neonatal intracranial injury. Obstet Gynecol 2011;118(6):1239-1246. https://doi. org/10.1097/aog.0b013e31823835d 3

17. Towner D, Castro MA, Eby-Wilkens E, Gilbert WM. Effect of mode of delivery in nulliparous women on neonatal intracranial injury. N Engl J Med 1999;341(23):1709-1714. https://doi.org/10.1056/ nejm199912023412301

18. Allen VM, O'Connell CM, Farrell SA, Baskett TF. Economic implications of method of delivery. Am J Obstet Gynecol 2005;193(1):192-197. https://doi.org/10.1016/j.ajog.2004.10.635

19. Peters H, Francis K, Harding K, Tookey PA, Thorne C. Operative vaginal delivery and invasive Peters $\mathrm{H}$, Francis K, Harding K, Tookey PA, Thorne C. Operative vaginal delivery and invasive
procedures in pregnancy among women living with HIV. Eur J Obstet Gynecol Reprod Biol procedures in pregnancy among women living with HIV.

20. Royal College of Obstetricians and Gynaecologists. Operative Vaginal Delivery. London: RCOG, 2011. https://www.rcog.org.uk/en/guidelines-research-services/guidelines/gtg26/ (accessed 9 June 2017).

21. Ismail NAM, Saharan WSL, Zaleha MA, Jaafar R, Muhammad JA, Razi ZRM. Kiwi Omnicup versus Malmstrom metal cup in vacuum assisted delivery: A randomized comparative trial. J Obstet Gynaecol Res 2008;34(3):350-353. https://doi.org/10.1111/j.1447-0756.2007.00701.x

22. Mola GDL, Kuk JM. A randomised controlled trial of two instruments for vacuum-assisted delivery (Vacca Re-Usable Omnicup and the Bird anterior and posterior cups) to compare failure rates, safety and use effectiveness. Aust N Z J Obstet Gynaecol 2010;50(3):246-252. https://doi.org/10.1111/j.1479$828 \mathrm{x} .2010 .01166 . \mathrm{x}$

Accepted 26 September 2017 